\title{
Visualizing the multifractal wave functions of a disordered two-dimensional electron gas
}

\author{
Berthold Jäck $\odot,{ }^{1,2}$ Fabian Zinser, ${ }^{1}$ Elio J. König $\odot,{ }^{1,3}$ Sune N. P. Wissing, ${ }^{4}$ Anke B. Schmidt $\odot,{ }^{4}$ Markus Donath $\odot,{ }^{4}$ \\ Klaus Kern, ${ }^{1,5}$ and Christian R. Ast $\odot^{1}$ \\ ${ }^{1}$ Max-Planck-Institut für Festkörperforschung, 70569 Stuttgart, Germany \\ ${ }^{2}$ Princeton University, Joseph Henry Laboratories and Department of Physics, Princeton, New Jersey 08544, USA \\ ${ }^{3}$ Rutgers University, Department of Physics, Piscataway, New Jersey 08854, USA \\ ${ }^{4}$ Physikalisches Institut, Westfälische Wilhelms-Universität Münster, 48149 Münster, Germany \\ ${ }^{5}$ Institut de Physique, Ecole Polytechnique Fédérale de Lausanne, 1015 Lausanne, Switzerland
}

(Received 20 August 2020; accepted 14 December 2020; published 8 January 2021)

\begin{abstract}
The wave functions of a disordered two-dimensional electron gas at the quantum-critical Anderson transition are predicted to exhibit multifractal scaling in their real space amplitude. We experimentally investigate the appearance of these characteristics in the spatially resolved local density of states of the two-dimensional mixed surface alloy $\mathrm{Bi}_{\mathrm{x}} \mathrm{Pb}_{(1-\mathrm{x})} / \mathrm{Ag}(111)$, by combining high-resolution scanning tunneling microscopy with spin- and angle-resolved inverse-photoemission experiments. Our detailed knowledge of the surface alloy's electronic band structure, the exact lattice structure, and the atomically resolved local density of states enables us to construct a realistic Anderson tight binding model, and to directly compare the measured local density of states characteristics with those from our model calculations. The statistical analyses of these two-dimensional local density of states maps reveal their log-normal distributions and multifractal scaling characteristics of the underlying wave functions with a finite anomalous scaling exponent. Finally, our experimental results confirm theoretical predictions of an exact scaling symmetry for Anderson quantum phase transitions in the Wigner-Dyson classes.
\end{abstract}

DOI: 10.1103/PhysRevResearch.3.013022

\section{INTRODUCTION}

Multifractality is an ubiquitous phenomenon in nature that characterizes a variety of physical phenomena from turbulence [1] to fluid flow and surface growth [2]. It describes the power-law scaling of fractal quantities with not one but a continuous (infinite) set of anomalous scaling exponents. Multifractality also governs the physics of the Anderson quantum phase transition in disordered systems [3,4], which separates phases with Anderson localized [5] and extended wave functions in insulators and metals, respectively. In this sense, Anderson transitions are substantially richer than ordinary quantum phase transitions, where the set of anomalous scaling exponents is finite.

At Anderson criticality, multifractality characterizes the real space amplitude fluctuations of the underlying quantum mechanical wave functions $\Psi(\vec{r})[6]$ and thereby the real space distribution of the corresponding local electronic density of states (LDOS) [7,8]. Geometrically, it accounts for the spectrum of fractal dimensions associated with topographic lines in the real space distribution of $|\Psi(\vec{r})|^{2}$, as illustrated in Fig. 1(a). Defining such lines by $|\Psi(\vec{r})|^{2}=$ const. $\times L^{-\alpha}$

Published by the American Physical Society under the terms of the Creative Commons Attribution 4.0 International license. Further distribution of this work must maintain attribution to the author(s) and the published article's title, journal citation, and DOI.
( $L$ is the system size) $[9,10]$ the probability distribution for the observation of a real space LDOS scaling at a given singularity strength $\alpha$ is called the "singularity spectrum", $f(\alpha)$. [9,11,12]; i.e., by changing the system size $L,|\Psi(\vec{r})|^{2}$ scales with the same exponent along one topographic line. In the presence of multifractality at the Anderson transition, $f(\alpha)$ describes a continuous set of scaling exponents, which, away from the quantum critical point in the metallic phase, collapses onto a line for scaling with a single exponent [Fig. 1(b)].

Scanning tunneling microscopy (STM) experiments can probe the LDOS with atomic resolution, and they are particularly useful to study wave function multifractality of twodimensional (2D) systems. Previous STM experiments on disordered two-dimensional quantum Hall systems [13,14], on the surface of bulk GaAsMn [15], and on 2D layers of transition metal dichalcogenides [16] reported $f(\alpha)$ spectra, calculated from differential tunneling conductance, $d I / d V$, maps, whose broadened characteristics were consistent with an interpretation of multifractal scaling at criticality [cf., Fig. 1(b)].

However, such $d I / d V$ maps do not necessarily reflect the pure LDOS, but commonly contain contribution from other phenomena such as charge density waves [17], quasiparticle interference [16], and electronic correlation [15], thereby obscuring the underlying eigenstate distribution characteristics. Without a sufficiently large measurement window [13] or full access to the wave-function decay in the three-dimensional bulk [15], a complete picture of the eigenstate real space decay cannot be captured. Since little is known about the underlying 

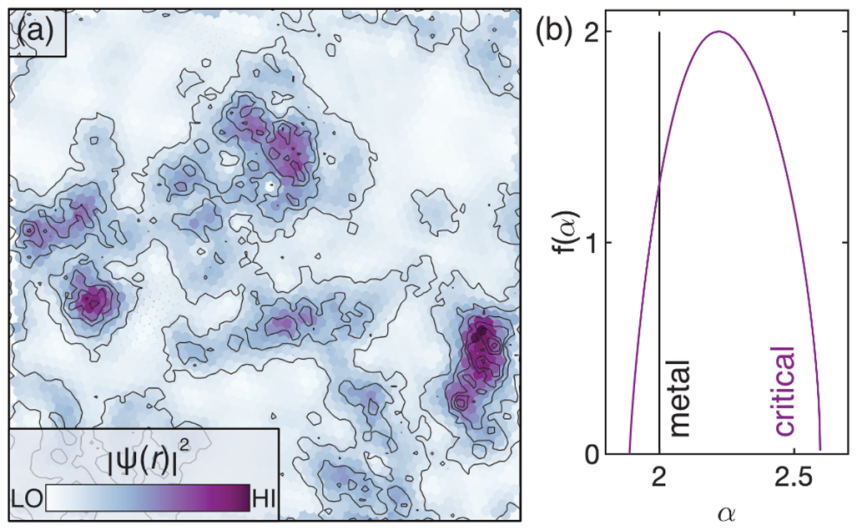

FIG. 1. Multifractal wave-function scaling in two dimensions. (a) Calculated LDOS $\left(|\Psi(\vec{r})|^{2}\right)$ map (approx. $50 \times 50$ lattice sites) of a two-dimensional electron gas exhibiting multifractal scaling behavior with power-law decay characteristics. The LDOS scales with different exponents $\alpha$ along different topographic lines (black solid lines). (b) Schematic plot of the singularity spectrum $f(\alpha)$ for a $d=2$ dimensional system with multifractal (purple) and metallic (black) scaling behavior.

local electronic structure in the presence of disorder in most material systems studied to date, experimental results cannot be compared with theoretical expectations, which all together render the interpretation of a multifractal analysis challenging.

In this article, we experimentally investigate the LDOS of the $2 \mathrm{D}$ mixed surface alloy $\mathrm{Bi}_{x} \mathrm{~Pb}_{1-x} \mathrm{Ag}$ and report multifractal wave function scaling in a disordered $2 \mathrm{D}$ electron gas with strong Rashba spin-orbit coupling (SOC). To this end, we combine high-resolution STM measurements with inversephotoemission experiments (IPE). This combined approach provides us with detailed knowledge on the local electronic properties and the atomic scale lattice structure of the disordered surface alloy with chemical contrast. On this basis, we construct a realistic Anderson tight binding model, which allows us to compare the measured $d I / d V$ map characteristics with the calculated LDOS maps. The quantitative analyses of these maps reveal their log-normal distributions and the multifractal scaling of the mixed surface alloy LDOS. Finally, our experimental results are in agreement with theoretical expectations of an exact symmetry of the scaling exponents for Anderson quantum phase transitions in the Wigner-Dyson classes.

\section{RESULTS}

The $\mathrm{Bi}_{x} \mathrm{~Pb}_{1-x} / \mathrm{Ag}(111)$ mixed surface alloy is a $2 \mathrm{D}$ alloy of randomly mixed bismuth, $\mathrm{Bi}$, and lead, $\mathrm{Pb}$, atoms on the (111) facet of a silver, Ag, surface [18]. The atomically resolved STM topography of the surface alloy is shown in Fig. 2(a). It visualizes the random arrangement of $\mathrm{Bi}$ and $\mathrm{Pb}$ atoms on the triangular $\sqrt{3} \times \sqrt{3} \mathrm{R} 30^{\circ}$ alloy lattice, where they substitute every third Ag atom of the surface layer, leading to strong compositional disorder at any mixing ratio without clustering [19]. The $\mathrm{Bi}$ and $\mathrm{Pb}$ atoms retain their individual relaxation position outside the plane of the $\operatorname{Ag}(111)$ surface plane [Fig. 2(b)] by which they can be distinguished in terms of their topographic height contrast, $\Delta z$, in STM topography maps [20]. Such analysis applied to the topography shown in Fig. 2(a) is illustrated by the histogram in Fig. 2(c) and reveals a $\mathrm{Bi}_{0.79} \mathrm{~Pb}_{0.21} / \mathrm{Ag}(111)$ mixing ratio.

The 2D electronic structure of this type of surface alloys is characterized by a set of three bands, $s p_{\mathrm{z}}, m_{j}=1 / 2$, and $m_{j}=3 / 2$ [18]. We performed inverse-photoemission experiments on the $\mathrm{Bi} / \mathrm{Ag}(111)\left(\mathrm{Bi}_{x} \mathrm{~Pb}_{1-x} / \mathrm{Ag}(111), x=1\right)$ surface alloy to characterize the band dispersions along the $\bar{\Gamma} \overline{\mathrm{K}}$ direction (see Appendix A) [21]. The measured dispersions of the unoccupied $m_{j}=1 / 2$ and $m_{j}=3 / 2$ bands shown in Fig. 2(d) are consistent with results from $a b$ initio calculations for that platform [18]; please refer to Ref. [22] for the photo-emission data of the occupied $s p_{\mathrm{z}}$ band in previous work. The band structure is visibly affected by strong SOC [22], and it is confined within the first atomic layer at the sample surface [22], evidencing the $2 \mathrm{D}$ character of the surface alloy's electronic properties.

Complete substitution of $\mathrm{Bi}$ atoms by $\mathrm{Pb}$ atoms in their lattice sites results in the formation of the pure $\mathrm{Pb} / \mathrm{Ag}(111)$ surface alloy $\left(\mathrm{Bi}_{x} \mathrm{~Pb}_{1-x} / \mathrm{Ag}(111), x=0\right)$. Owing to the different number of valence electrons in $\mathrm{Bi}\left(6 s^{2} 6 p^{3}\right)$ and $\mathrm{Pb}$ $\left(6 s^{2} 6 p^{2}\right)$, the bands of a pristine $(x=0) \mathrm{Pb} / \operatorname{Ag}(111)$ alloy $\left(s p_{\mathrm{z}}\right.$ onset at $E \approx 650 \mathrm{meV}$ ) will shift by about $0.8 \mathrm{eV}$ to lower energy, when going to a pristine $(x=1) \mathrm{Bi} / \mathrm{Ag}(111)$ alloy ( $s p_{\mathrm{z}}$ onset at $E \approx-135 \mathrm{meV}$ ) $[18,23]$. The partial substitution of $\mathrm{Bi}$ by $\mathrm{Pb}$ in the mixed surface alloy $\mathrm{Bi}_{0.79} \mathrm{~Pb}_{0.19} / \mathrm{Ag}(111)$, therefore, results in a random disorder potential, whose spatial characteristics are determined by the position of the $\mathrm{Pb}$ atoms on the alloy lattice [Fig. 2(a)].

Scanning tunneling spectroscopy measurements can be used to characterize the influence of this random disorder potential on the LDOS. Figure 2(e) shows selected $d I / d V$ spectra, which were recorded in different regions of the sample surface, exhibiting a varying local Bi concentration [cf., Fig. 2(a)]. The spectra, all of which were recorded with the STM tip located on top of a $\mathrm{Bi}$ atom, exhibit similar spectral features that can be associated with the onsets of the respective electronic bands [cf., Fig. 2(d)]. However, we find the energetic position of these peaks to be shifted with respect to each other in the different tunneling spectra; their positions shift from higher to lower energy, when moving from regions of locally high $\mathrm{Pb}$ concentration to regions of locally high $\mathrm{Bi}$ concentration [23], consistent with the presence of a locally varying electrostatic potential. In passing we note that the tunneling spectra also do not exhibit a suppression of spectral weight around the Fermi level typically associated with electron-electron interactions in the presence of disorder [15,24-27].

To capture the real space characteristics of these disorder induced LDOS variations, we have performed spectroscopic imaging with the STM, where we record $d I / d V$ maps over a larger surface area highlighted in Fig. 3(a) (see Appendix A). The normalized $d I / d V$ map measured at the $s p_{\mathrm{z}}$-band onset at $E=80 \mathrm{meV}$, Fig. 3(b), exhibits a highly irregular pattern with significant amplitude variations over nanometer length scales. We find that a high $d I / d V$ signal is predominantly accumulated in areas of high $\mathrm{Pb}$ concentration, consistent with the characteristics of the $d I / d V$ spectra in Fig. 2(e). Conversely, 

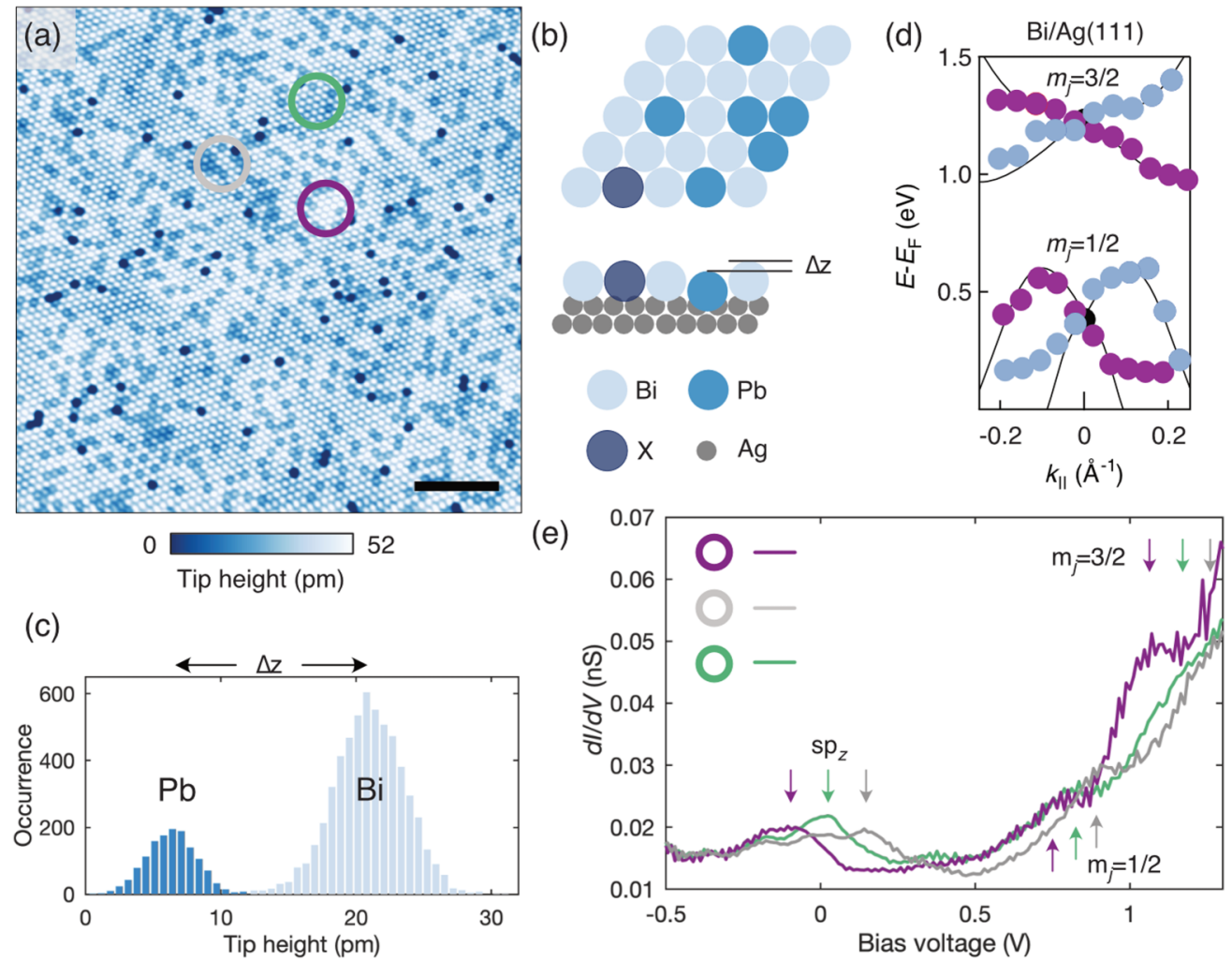

FIG. 2. Electronic properties and atomic lattice structure of the mixed surface alloy. (a) STM topography of the $\mathrm{Bi}_{0.79} \mathrm{~Pb}_{0.21} / \mathrm{Ag}(111)$ mixed surface alloy (setpoint $V=3 \mathrm{mV}, I=7 \mathrm{nA}$ ). Scale bar $5 \mathrm{~nm}$. (b) Schematic sketch of the mixed surface alloy's atomic lattice structure. The alloy atoms substitute every third Ag atom of the surface layer, forming a $\sqrt{3} \times \sqrt{3} \mathrm{R} 30^{\circ}$ lattice structure. $\Delta \mathrm{z}$ denotes the apparent height difference between the $\mathrm{Bi}$ and $\mathrm{Pb}$ atoms as measured with the $\mathrm{STM}$; X denotes an alloy lattice vacancy. (c) Histogram analysis of the topography in (a) displaying the occurrence of the relative STM tip apparent height. (d) Band structure of the Bi/Ag(111) surface alloy along $\overline{\Gamma K}$ measured by spin-resolved IPE. The data points indicate the peak positions with the solid lines as guides to the eye. Purple (blue) colors refer to the spin-quantization axis forming a right (left) handed coordinate system with the $\overline{\Gamma K}$ line, and the sample normal. (e) $d I / d V$ tunneling spectra (setpoint $V=-1 \mathrm{~V}, I=1 \mathrm{nA}, V_{\text {mod }}=25 \mathrm{mV}$ ) measured with the STM tip located at different surface locations, which are indicated in (a). The spectral features are labeled according to their band character.

the corresponding $d I / d V$ map measured deep inside the $s p_{\mathrm{z}}$ band at $E=-400 \mathrm{meV}$, Fig. 3(c), lacks a pronounced spatial structure, which is in agreement with the location-independent appearance of the individual $d I / d V$ spectra at this energy. We further find that the $d I / d V$ maps do not exhibit periodically ordered real space patterns, indicating the absence of charge density wave formation in the mixed surface alloy.

Knowledge of disorder induced LDOS characteristics from model calculations can help to reveal the origin of the irregular $d I / d V$ pattern near the $s p_{\mathrm{z}}$-band edge. We have constructed a realistic 2D Anderson tight binding model of the mixed surface alloy to calculate LDOS maps [5]. Commonly, numerical investigations of the Anderson transition in two dimensions rely on artificial disorder distributions for the calculation of the eigenstates [28,29]. In our study, we create a realistic Anderson model with a binary distribution of on-site disorder potentials, which is based on our detailed knowledge of the electronic properties and the alloy lattice structure from IPE and STM experiments, respectively. We obtain the nearestneighbor hopping potentials and Rashba terms from fits to the measured band dispersion in Fig. 2(d). Moreover, our ability to distinguish between $\mathrm{Bi}$ and $\mathrm{Pb}$ atoms in $\mathrm{STM}$ measurements [cf., Fig. 2(c)] enables us to extract the exact atomic lattice structure of the topography in Fig. 3(a). The reconstructed lattice is shown in Fig. 3(d); we use it as a structural model input to solve for the wave functions $\Psi(\vec{r})$ and to calculate realistic LDOS $|\Psi(\vec{r})|^{2}$ maps (see Appendix B). We also included a Rashba term, to account for the effect of strong SOC in the 2D mixed surface alloys [22,30].

The calculated LDOS map near the $s p_{\mathrm{z}}$-band edge is shown in Fig. 3(e). It is in very good agreement with the corresponding $d I / d V$ map, Fig. 3(b), down to the very detail. The measured and calculated maps deep inside the $s p_{\mathrm{z}}$ band, Figs. 3(c) and 3(f) respectively, exhibit a comparable amount of agreement despite their rather featureless appearance.

\section{DISCUSSION}

The remarkable agreement between the $d I / d V$ maps and LDOS maps shows that the measured $d I / d V$ signal predominantly reflects the spatial distribution of the $2 \mathrm{D}$ mixed surface alloy LDOS, with contributions coming from the $\mathrm{Bi}$ and $\mathrm{Pb}$ atoms only. This agreement is not least promoted by the absence of signatures of electronic correlations and charge density wave order in our experimental data in Figs. 2(e) and 2(b)-2(c), respectively. While the absence of these 

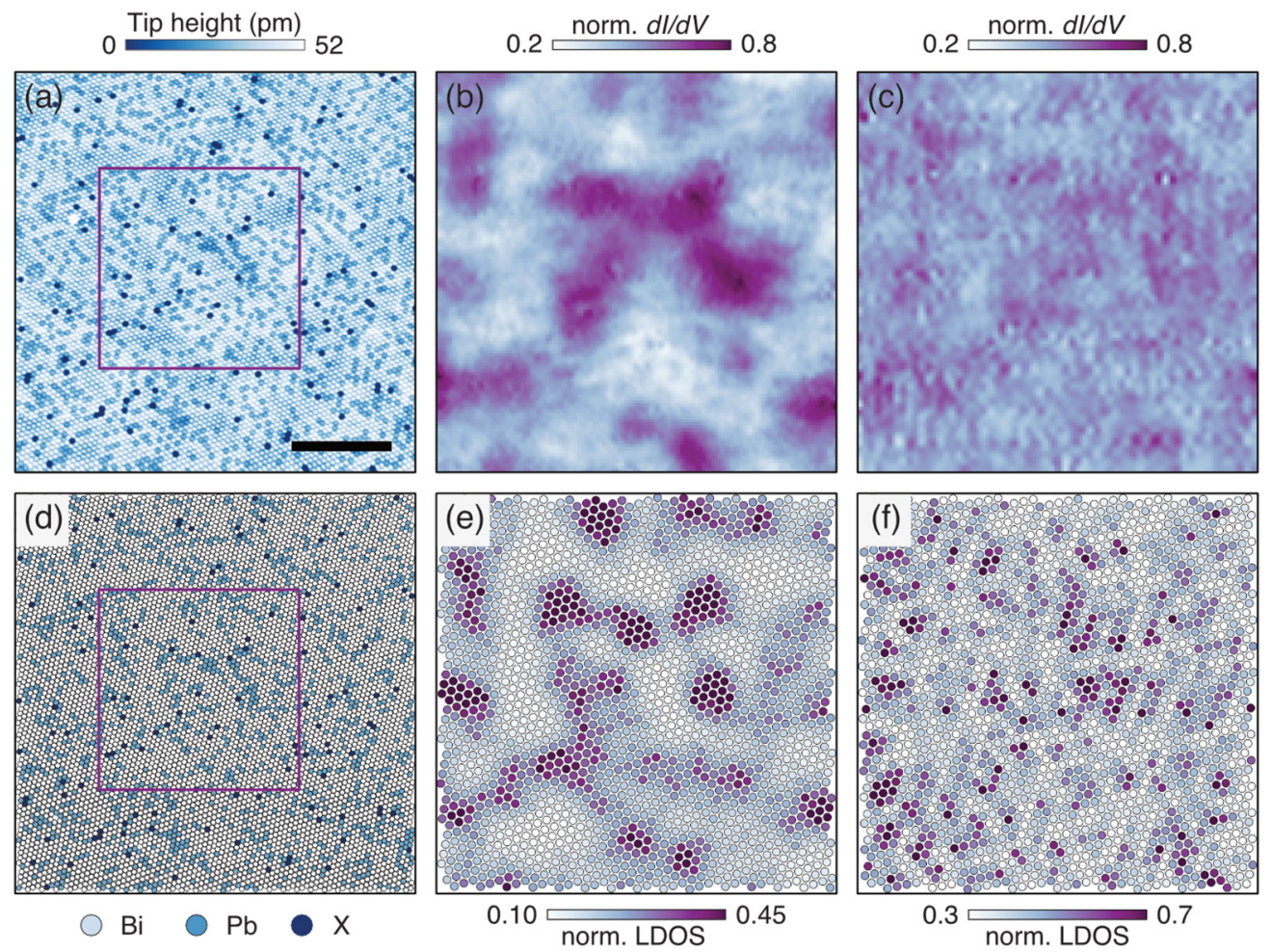

FIG. 3. (a) STM topography of the $\mathrm{Bi}_{0.79} \mathrm{~Pb}_{0.21} / \mathrm{Ag}(111)$ mixed surface alloy (setpoint $V=3 \mathrm{mV}, I=7 \mathrm{nA}$ ). Scale bar $10 \mathrm{~nm}$. The purple box $\left(20 \times 20 \mathrm{~nm}^{2}\right)$ highlights the area in which the normalized $d I / d V$ maps have been measured: (b) at the $s p_{\mathrm{z}}$-band edge $(+0.08 \mathrm{eV})$, (c) within the $s p_{\mathrm{z}}$ band $(-0.4 \mathrm{eV})$ (setpoint $I=1 \mathrm{nA}, V_{\text {mod }}=25 \mathrm{mV}$ ). (d) Reconstructed lattice model of the mixed surface alloy, where the topography in (a) serves as structural input for the Anderson tight-binding model calculation. Panel (e) and (f) show the calculated normalized LDOS maps at the $s p_{\mathrm{z}}$-band edge and inside the $s p_{\mathrm{z}}$ band, respectively (please refer to Table I for numerical parameters).

phenomena could be explained by the elevated temperature of our experiment $(100 \mathrm{~K})$, it is also consistent with the metallic character and the relatively simple topology of the $s p_{z}$ band [cf., Fig. 2(d)] [18].

Deviations between the measured and calculated spatial LDOS distributions likely result from the simplicity of our Anderson tight binding model described in Appendix B. This model considers only isotropic nearest-neighbor hopping terms within a single band. It further neglects coupling between alloy atoms and $\mathrm{Ag}$ substrate, which should directly influence the electronic structure, and which we identify as a major source of these deviations. We anticipate that an extension of the model by next-nearest-neighbor terms would, by contrast, cause comparably smaller corrections to the electronic structure of the mixed surface alloy and can, therefore, be neglected. This choice is, further, justified by the good agreement between the experimentally determined, see Fig. 2(d), and calculated, Fig. 5, free-electron-like $s p_{\mathrm{z}}$-band structure, respectively.

The agreement between experiment and theory, therefore, emphasizes the predominant influence of the $\mathrm{Pb}$ atom induced random disorder potential on the spatial distribution of the mixed surface alloy's LDOS. This finding is also reflected in the individual $d I / d V$ spectra in Fig. 2(e), where the local $\mathrm{Pb}$ concentration affects the energetic position of the $s p_{\mathrm{z}}$-band edge. Consequently, these spectra miss the sharp Rashba-induced van-Hove singularity at the band onset, which was previously observed for both the pure $\mathrm{Bi} / \mathrm{Ag}(111)$ and $\mathrm{Pb} / \mathrm{Ag}(111)$ surface alloys [23]. Importantly, we emphasize that the characteristic length scale of the measured and calculated LDOS near the band edge, see Figs. 3(b) and 3(e) respectively, is much larger than the atomic length scale of the disorder potential. The LDOS does not merely follow the local potential; instead, its structure emerges from the antagonistic interplay of localizing $\mathrm{Pb}$ impurities and delocalizing kinetic energy.

Theoretically, for binary alloys, a disorder induced exponential tail in the average density of states is expected inside the (clean) band gap [31]. This tail is formed by spatially localized impurity states, which (for the present case of a $2 \mathrm{D}$ system with spin-orbit coupling) are energetically separated by a sharp mobility edge from extended states far inside the band [32]. This picture is corroborated by our detailed analysis of the wave function decay characteristics based on the normalized partition ratio, NPR [33]: We find that the average wave-function decay length is of atomic scale at the $s p_{\mathrm{z}}$-band edge, and reaches the size of our measurement frame $(20 \mathrm{~nm})$ at $E \approx-100 \mathrm{meV}$ (see Appendix D). The picture of a mobility edge also manifests in the spatial characteristics of the LDOS distributions; while the LDOS inside the localized impurity band and near the mobility edge exhibits a spatially irregular pattern, Figs. 3(b) and 3(e), the LDOS deep inside the the $s p_{\mathrm{z}}$ band, Figs. 3(c) and 3(f), appears more homogeneous, characteristic for the extended wave function of a metal.

These observations can be further quantified by investigating the LDOS distribution function, which illustrates the 

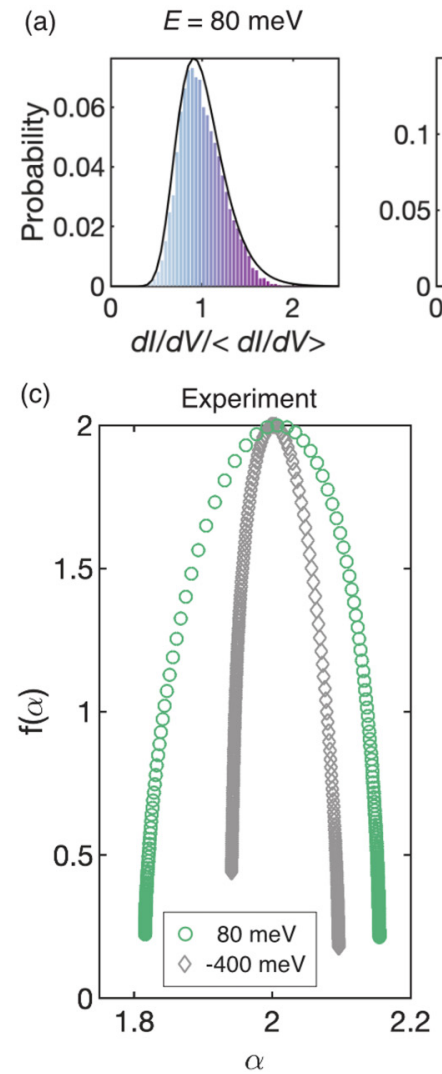

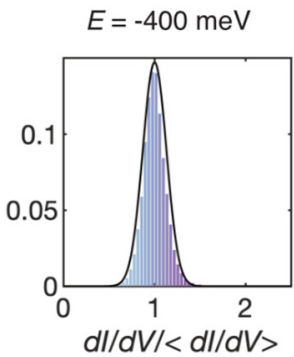

(d)

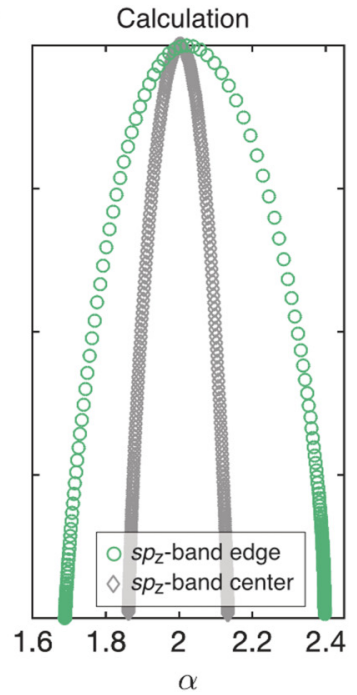

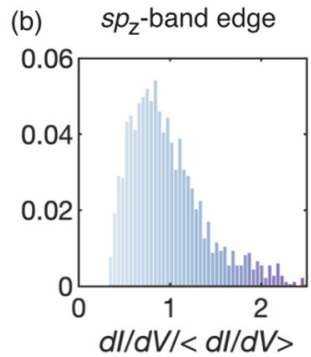

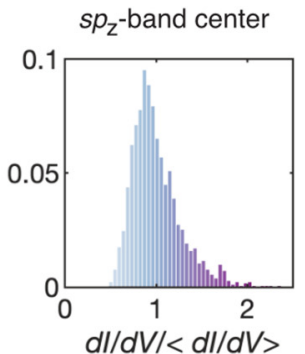

(e)
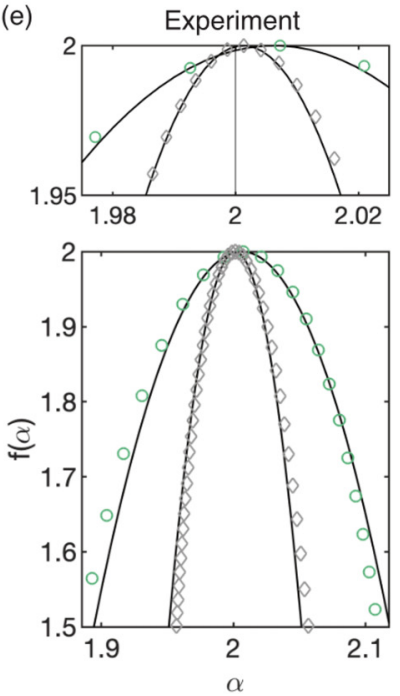

FIG. 4. Multifractal analysis of the measured and calculated LDOS maps. (a) Left panel: Statistical distribution of the $d I / d V$ amplitude for the map measured at $V=80 \mathrm{mV}$ near the $s p_{\mathrm{z}}$-band edge in Fig. 3(b). Right panel: The same analysis applied to the map measured at $V=-400 \mathrm{mV}$ inside the $s p_{\mathrm{z}}$ band in Fig. 3(c). The black lines represent the corresponding calculated distribution functions. (b) Left panel: Statistical distribution of the LDOS amplitude for the map calculated at the $s p_{\mathrm{z}}$-band edge in Fig. 3(e). Right panel: The same analysis applied to the calculated map inside the $s p_{\mathrm{z}}$-band in Fig. 3(f). (c) $f(\alpha)$ of the $d I / d V$ maps in Fig. 3(b) (purple circles) and Fig. 3(c) (blue diamonds). (d) $f(\alpha)$ of the calculated LDOS maps in Fig. 3(e) (purple circles) and Fig. 3(f) (blue diamonds). (e) Bottom panel: Magnification of the $f(\alpha)$ spectra shown in (c). The black solid lines are the quadratic fits to the data. Top panel: Magnified view around the $f(\alpha)$ maxima.

relative occurrence of LDOS amplitudes in a given area [7,8]. Whereas the LDOS of extended wave functions follows a Gaussian distribution, wave function multifractality yields a log-normal LDOS distribution, anticipating the broad distribution of the insulating state [9]. Applying such an analysis to the measured LDOS maps of the $s p_{z}$ band, Figs. 3(b) and 3(c), reveals their log-normal characteristics near the band edge, $\mu=\log (0.975)$ and $\sigma=0.25)$, and, by contrast, their Gaussian characteristics, $\sigma=0.13$, inside the band [Fig. 4(a)]. We obtain similar characteristics for the LDOS distribution from model calculations, Fig. 4(c). Even though both LDOS maps follow a log-normal distribution, their skewness is more pronounced near the $s p_{\mathrm{z}}$-band edge as compared to inside the $s p_{\mathrm{z}}$ band, Fig. $4(\mathrm{c})$.

The corresponding multifractal singularity spectrum, $f(\alpha)$, can be directly calculated from the LDOS maps as a function of the singularity strength, $\alpha$, see Appendix C [34]. In Fig. 4(c) we plot $f(\alpha)$ of the measured LDOS maps. The broadened parabolic appearance of $f(\alpha)$, centered at $\alpha>2$, supports that the mixed surface alloy LDOS exhibits multifractal scaling behavior. $f(\alpha)$ of the LDOS map near the $s p_{\mathrm{z}}$-band edge, Fig. 4(c), is significantly wider than the $f(\alpha)$ of the LDOS map measured inside the $s p_{\mathrm{z}}$ band. This observation is consistent with the different character of the LDOS distribution functions in Fig. 4(a) and with the highly irregular (homogeneous) real space pattern of the LDOS maps in Figs. 3(b) [Fig. 3(c)]. $f(\alpha)$ of the calculated LDOS maps, Fig. 4(d), exhibit a wider shape than their experimental counterparts, consistent with the corresponding deviations between the LDOS distributions, Figs. 4(a) and 4(b), but show overall very good qualitative agreement. The deviations could, on the one hand, be rooted in the discussed simplifications of the Anderson model. On the other hand, the finite temperature of our experiment $(100 \mathrm{~K})$ promotes electron-phonon scattering induced quantum decoherence, weakening the eigenstate multifractality in the measured LDOS maps.

The anomalous eigenstate scaling distinguishes the quantum critical wave functions from those in the metallic phase at $\alpha=2$ [10]. It has been theoretically shown that the anomalous scaling of the Wigner-Dyson classes exhibits an exact symmetry [35], which, for a 2D system, manifests in the relation, $f(\alpha)=f(4-\alpha)-2+\alpha$. At weak multifractality, the associated singularity spectrum, $f(\alpha)=2-(\alpha-2-\gamma)^{2} /(4 \gamma)$, is parabolic, where $\gamma$ is a measure for the strength of multifractality and defines both the maximum at $\alpha=2+\gamma$ and the opening angle of the parabola [10]. We note that the mixed surface alloy, which preserves time-reversal symmetry and whose electronic states are strongly influenced by SOC 
[18,22], falls into the symplectic Wigner-Dyson class AII for which numerical calculations predict $\gamma=0.172$ [10].

Our quantitative analysis of the experimental $f(\alpha)$ in Fig. 4(e) reveals their predominently parabolic characteristics and consistency with the theoretical expectation of an exact anomalous scaling symmetry. Applying a quadratic fit to $f(\alpha)$ we obtain $\gamma_{\exp }=(6.3 \pm 0.4) \times 10^{-3}\left(\gamma_{\text {calc }}=\right.$ $\left.(21.6 \pm 1.1) \times 10^{-3}\right)$ and $\gamma_{\exp }=(1.30 \pm 0.05) \times 10^{-3}$ $\left(\gamma_{\text {calc }}=(2.60 \pm 0.05) \times 10^{-3}\right)$ for the experimental (calculated) LDOS map near the edge and inside the $s p_{\mathrm{z}}$ band. We note that nonparabolic corrections for $f(\alpha)<1.5$, Figs. 4(c) and 4(d), are in agreement with previous theoretical work on the scaling symmetry [29].

The extracted small values of $\gamma$, on the one hand, reflect moderate multifractality below the theoretically expected extent. It is likely that the previously discussed finite experimental temperature $(100 \mathrm{~K})$ weakens the multifractal characteristics. What is more, our measurement also integrates the eigenstates over a $70-\mathrm{meV}$ window, including those states deeper inside the band, which exhibit extended state characteristics; cf. normalized partition ratio analysis in Appendix D. In particular, the normalized disorder strength in the mixed surface alloy $W / t=0.7$ ( $W$ is the difference between $\mathrm{Bi}$ and $\mathrm{Pb}$ onsite potentials, $t$ is the nearest-neighbor hopping) such that most eigenstates in the mixed surface alloy $s p_{z}$ band are delocalized and away from the critical point. By comparison, highly accurate model calculations at criticality with $W / t>5$ show an enhancement of $\gamma$ by almost a factor of 10 that manifests in an accordingly larger degree of wave-function multifractality $[28,29]$.

Finally, in order to estimate the impact of the mentioned experimental limitations, we analyze the $d I / d V$ map measured at $E=1 \mathrm{eV}$ near the $m_{\mathrm{j}}=3 / 2$-band onset. Here one would also expect multifractal wave function characteristics similar to those observed near the $s p_{\mathrm{z}}$-band edge, Figs. 3(b) and 4(a), but these characteristics are not detected, Appendix E. Even though the real space pattern of the $d I / d V$ map, Fig. 7(a), appears irregular, areas of high and low $d I / d V$ amplitude, respectively, are found with similar occurrence; this is also reflected in the quasi-Gaussian distribution in Fig. 7(b). This discrepency of observations can be understood in terms of Fermi liquid theory, according to which quasiparticle scattering is strongly enhanced at $1 \mathrm{eV}$ away from the Fermi energy. This process promotes eigenstate decoherence, which is detrimental to the observation of multifractality characteristics. Argumentum a contrario, we take this discussion as evidence for sufficient quantum coherence for the detection of multifractality at the $s p_{\mathrm{z}}$ edge.

\section{CONCLUSION}

We combined STM and IPE experiments with Anderson model calculations to study the real space LDOS characteristics of the 2D mixed surface alloy $\mathrm{Bi}_{0.79} \mathrm{~Pb}_{0.21} / \mathrm{Ag}(111)$. Spectroscopic measurements of the LDOS with the STM reveal a strong influence of a locally varying disorder potential on the electronic states that is induced by the different valency of the $\mathrm{Bi}$ and $\mathrm{Pb}$ atoms.

The effect of disorder-induced wave function localization in the mixed surface alloy is quantified by the statistical analysis of the spatial LDOS distributions. We observe the log-normal characteristics of localized states near the $s p_{z^{-}}$ band edge, and the Gaussian characteristics of metallic states inside the $s p_{\mathrm{z}}$ band, indicating the presence of a mobility edge. The singularity spectra further reveal multifractal scaling of the eigenstate amplitude, which we find to be significant near the $s p_{\mathrm{z}}$-band edge.

We can reproduce these observations with very good agreement through realistic Anderson model calculations, which are based on our detailed knowledge of the electronic properties and the atomic scale lattice structure of the surface alloy. The same analysis further confirms the symmetry of the anomalous eigenstate scaling at criticality in the WignerDyson classes, which was theoretically predicted.

Looking ahead, realizing a larger disorder strength in this material system-for instance by moving beyond a binary alloy and by combining suitable elements with enhanced onsite potential differences-would be desirable, because such systems could provide deeper insights into strong multifractal eigenstate scaling $[10,29]$. We anticipate that performing these and related experiments at much reduced temperatures should suppress scattering-induced eigenstate decoherence and enhance the detection of multifractal characteristics. Recent experiments on 2D films of disordered transition-metal dichalcogenides also suggest the existence of multifractal superconductivity $[16,36]$. Applying our methodology to these systems could provide unique insights on the underlying normal state eigenstate multifractality and its correlation with the superconducting critical temperature [37-41]. In this regard, the actively studied material class of the superconducting high entropy alloys could represent avenues to explore the interplay of wave function multifractality at strong compositional disorder with the properties of the superconducting order parameter $[42,43]$.

\section{ACKNOWLEDGMENTS}

We gratefully acknowledge stimulating discussions with Maurits W. Haverkort, M. Assig, C. Straßer, M. Etzkorn, H. Benia, M. Eltschka, and A. Schnyder. B.J. acknowledges funding from the Alexander-von-Humboldt foundation during the completion of this work. E.J.K. acknowledges support by DOE Basic Energy Sciences Grant No. DEFG02-99ER45790. C.R.A. acknowledges funding from the Emmy-Noether-Program of the Deutsche Forschungsgemeinschaft (DFG).

\section{APPENDIX A: MATERIALS AND METHODS}

Preparation of the $\mathrm{Bi}_{x} \mathrm{~Pb}_{1-x} / \mathrm{Ag}(111)$ mixed surface alloys was done by the coevaporation of sub-monolayer amounts of $\mathrm{Bi}$ and $\mathrm{Pb}$ atoms on an atomically clean $\mathrm{Ag}(111)$ surface. The Ag substrate was cleaned by subsequent cycles of argon sputtering and thermal annealing. During the alloy deposition, the sample was kept at a temperature of $573 \mathrm{~K}$ for optimal mobility of the atoms to form a long-range ordered alloy. Sample preparation and measurements were done in the ultrahigh vacuum environment, $p \geqslant 10^{-10} \mathrm{mbar}$, of a home-built scanning tunneling microscope. The temperature of tip and sample during measurements was $100 \mathrm{~K}$. The tunnel 
conductance spectra and maps were measured at appropriate bias $V$ and current $I$ setpoints, respectively, which are indicated in the figure captions, using a lock-in modulation amplitude, $V_{\text {mod }}=25 \mathrm{mV}$. A flat background was subtracted from the tunnel conductance map to account for the finite and spatially homogeneous background LDOS from the $\mathrm{Ag}(111)$ bulk states. The overall energy resolution of our experiment was determined to be about $70 \mathrm{meV}$.

Spin-resolved IPE was performed at room temperature using spin-polarized electrons emitted from a GaAs photocathode [44]. The photons were detected with a Geiger-Müller counter at $E=9.8 \mathrm{eV}$ [45]. The overall IPE energy resolution was $350 \mathrm{meV}$ and the angular resolution $\pm 2^{\circ}$ [46].

\section{APPENDIX B: ANDERSON TIGHT BINDING MODEL}

We calculate theoretical LDOS maps using a realistic Anderson tight binding model of the 2D mixed surface alloy $[5,47,48]$. We use a single $p_{z}$-orbital model with on-site potential $\epsilon_{\mathrm{i}}$ and a nearest-neighbor hopping potential $t$, which is isotropic on the alloy's 2D hexagonal lattice. To simplify the model calculation, we also assume identical hopping potentials for all different nearest-neighbor pairs and we neglect coupling of the alloy atoms to the Ag substrate. Strong SOC in the $\mathrm{Bi}_{x} \mathrm{~Pb}_{1-x} / \mathrm{Ag}(111)$ mixed surface alloy [22] is considered by a nearest-neighbor Rashba-type spin-splitting with coupling constant $\alpha_{R}$ [30,49]:

$$
H=\sum_{i, \sigma_{i}} \epsilon_{i}\left|i, \sigma_{i}\right\rangle\left\langle i, \sigma_{i}\left|+\sum_{\langle i, j\rangle, \sigma_{i}=\sigma_{j}} t\right| i, \sigma_{i}\right\rangle\left\langle j, \sigma_{j}\left|+\sum_{\langle i, j\rangle, \sigma_{i} \neq \sigma_{j}} \alpha_{R}\left(\vec{\sigma} \times \vec{\tau}_{i j}\right)_{z}\right| i, \sigma_{i}\right\rangle\left\langle j, \sigma_{j}\right| .
$$

The angle parentheses in the second sum limits indicate summation over nearest-neighbor, $\vec{\sigma}$ is the vector of Pauli matrices, $\vec{\tau}_{i j}$ is a unit vector connecting two neighboring atoms, and $\sigma_{i}$ is the spin index. We use the reconstructed atomic lattice structure in Fig. 3(d) as structural model input to assign the $\mathrm{Bi}$ and $\mathrm{Pb}$ specific on-site potentials $\epsilon_{\mathrm{i}}$. The tight-binding calculation gives access to the complete set of eigenstates $\Psi(\vec{r}, E)$ of the mixed surface alloy that can be used to calculate spatially resolved eigenstate density from eigenstate spinors,

$$
|\Psi(\vec{r}, E)|^{2}=|\Psi(\vec{r}, E, \uparrow)|^{2}+|\Psi(\vec{r}, E, \downarrow)|^{2} .
$$

Realistic LDOS maps [cf., Figs. 3(e) and 3(f)] are obtained by convolving the spatially resolved eigenstate densities with a Gaussian function $(\sigma=35 \mathrm{meV})$ to account for the finite temperature and energy resolution of our experiment.

We obtain realistic values for the Rashba and hopping potentials by fitting the calculated surface alloy bands to the experimentally determined band dispersions [Fig. 1(d)]. The band dispersion in momentum space can be calculated by a

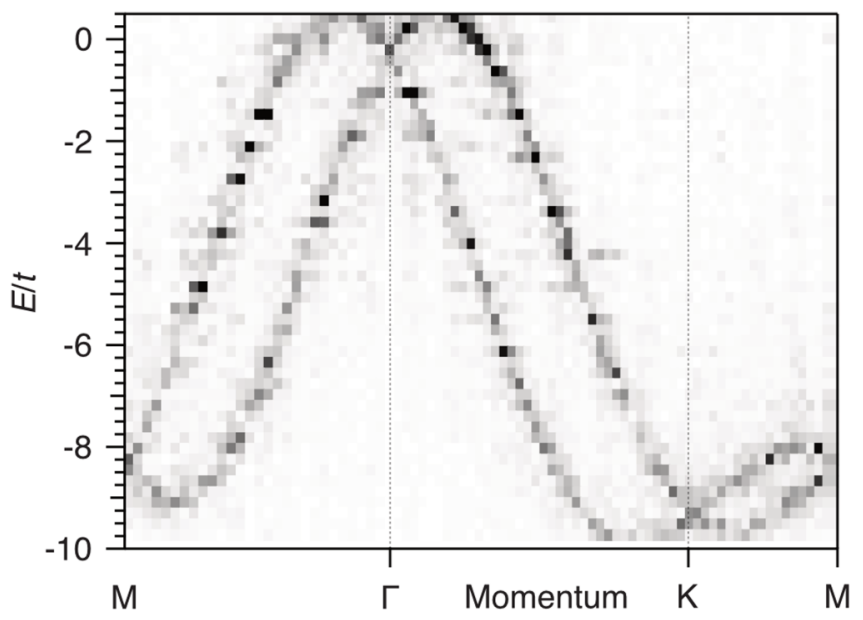

FIG. 5. Calculated band structure of the $s p_{\mathrm{z}}$ band. The corresponding tight-binding parameter are given in Table I. The energy scale is given in units of $E / t$.
Fourier transformation of the eigenstates obtained from the Anderson tight-binding model under periodic boundary conditions [50-53]. The calculated $s p_{\mathrm{z}}$-band dispersion, which is shown in Fig. 5, is consistent with results from $a b$ initio calculations [18] and from photoemission experiments [22]. Additionally, we find good agreement between the resulting hopping parameter, $t=0.4 \mathrm{eV}$, and the full $s p_{\mathrm{z}}$ bandwidth, $W \approx 3.5 \mathrm{eV}$, which was determined in photoemission experiments [54]. $W / t=8.75$ closely matches the bandwidth of the calculated $s p_{\mathrm{z}}$ band shown in Fig. 5.

Finally, we obtain a realistic estimate for the $\mathrm{Bi}$ and $\mathrm{Pb}$ on-site potential difference $\Delta \epsilon=\epsilon_{\mathrm{Pb}}-\epsilon_{\mathrm{Bi}}$ from the relative energy shift of the band onsets in the $d I / d V$ spectra in Fig. 2(e). The complete parameter set for the Anderson tightbinding model is given in Table I.

\section{APPENDIX C: MULTIFRACTAL ANALYSIS}

The multifractal singularity spectrum $f(\alpha)$ can be calculated from 2D maps using a method developed by Chhabra and Jensen [34]. This analysis is based on the numerical evaluation of the normalized local box probabilities $\mu_{\mathrm{j}}(\lambda, q)$,

$$
\mu_{j}(\lambda, q)=\frac{1}{\sum_{k=1}^{N(\lambda)} P_{k}(\lambda)^{q}} P_{j}(\lambda)^{q},
$$

TABLE I. Parameters used in the tight-binding calculation for the different local density of states (LDOS) maps. The first column indicates the band character. $E_{\exp }$ is the energy at which the experimental LDOS map has been measured, $\epsilon_{\mathrm{Bi}}$ and $\epsilon_{\mathrm{Pb}}$ are the on-site potentials for the $\mathrm{Bi}$ and $\mathrm{Pb}$ atoms, respectively. $\epsilon_{\mathrm{def}}$ is the on-site potential of an defect site, where no alloy atom is substituted (black dots in the topography). $t$ is the hopping parameter, $\alpha_{R}$ is the Rashba parameter, $E_{\mathrm{pos}}$ is the energy shift from the band edge at which the LDOS map has been calculated, and $E_{\text {broad }}$ is the full width at half maximum value for the Gaussian broadening with which the calculated data has been convoluted. All values are given in electronvolts.

\begin{tabular}{lrccccccc}
\hline \hline Band & $E_{\text {exp }}$ & $\epsilon_{\mathrm{Bi}}$ & $\epsilon_{\mathrm{Pb}}$ & $\epsilon_{\mathrm{def}}$ & $t$ & $\alpha_{R}$ & $E_{\mathrm{pos}}$ & $E_{\text {broad }}$ \\
\hline$s p_{\mathrm{z}}$ & 0.08 & -0.14 & 0.14 & 0.32 & 0.4 & 0.3 & -0.06 & 0.07 \\
$s p_{\mathrm{z}}$ & -0.40 & -0.14 & 0.14 & 0.32 & 0.4 & 0.3 & -0.6 & 0.07 \\
\hline \hline
\end{tabular}




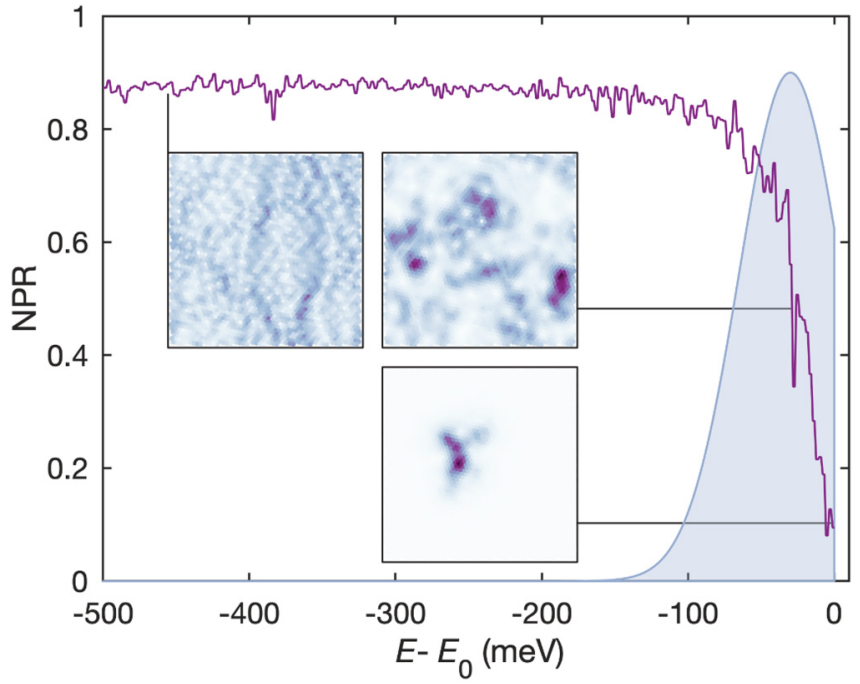

FIG. 6. NPR as a function of the energy, $E$, from the band onset, $E_{0}=0$, for each calculated eigenstate in the $s p_{\mathrm{z}}$ band. The shaded Gaussian shows the weighted integration interval for the calculation of the LDOS map in Fig. 3(e). The insets show the spatially resolved eigenstate densities, $|\Psi(\vec{r}, E)|^{2}$, at the respective energies (purple/white: high/low values, edge length $20 \mathrm{~nm}$ ).

where $P_{\mathrm{k}}(\lambda)^{q}$ denotes the probability of the qth moment of the LDOS distribution to be located in box $k$. The square shaped box with edge length, $L_{\mathrm{k}}$, is characterized by its normalized size, $\lambda=L_{\mathrm{k}} / L_{\mathrm{m}}$, determining the amount of boxes, $N$, inside a given square shaped LDOS map of edge length $L_{\mathrm{m}}$. Put into the context of the LDOS maps, $P_{\mathrm{k}}(\lambda)^{q}$ denotes the sum of the LDOS spectral weight inside a box of size $\lambda$ at position $k$.

Knowledge of $\mu_{\mathrm{j}}(\lambda, q)$ enables the direct calculation of $f(\alpha)$,

$$
f(\alpha(q))=\lim _{\lambda \rightarrow 0} \frac{\sum_{j=1}^{N(\lambda)} \mu_{j}(\lambda, q) \ln \left(\mu_{j}(\lambda, q)\right)}{\ln (\lambda)},
$$

and the singularity strength, $\alpha(q)$,

$$
\alpha(q)=\lim _{\lambda \rightarrow 0} \frac{\sum_{j=1}^{N(\lambda)} \mu_{j}(\lambda, q) \ln \left(P_{\mathrm{j}}(\lambda)^{q}\right)}{\ln (\lambda)} .
$$

We used $\lambda=0.1$ and a moment interval $q=[-200,200]$ for the calculation of the $f(\alpha)$ shown in Figs. 4(c) and 4(d).

\section{APPENDIX D: NORMALIZED PARTITION RATIO ANALYSIS}

The normalized partition ratio (NPR) can be used as a measure to quantify eigenstate localization in a given physical (a)
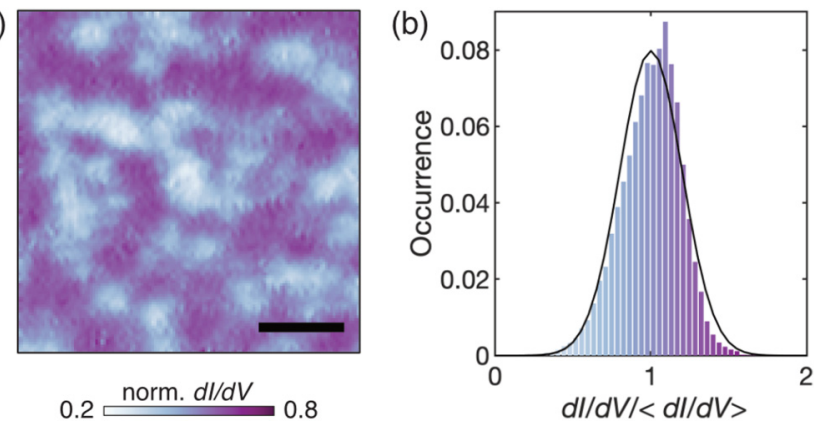

FIG. 7. (a) Normalized $d I / d V$ map [area: purple box in STM topography of Fig. 3(a)] measured around the band minimum of the $m_{\mathrm{j}}=3 / 2$-band (setpoint $V=1 \mathrm{~V}, I=1 \mathrm{nA}, V_{\text {mod }}=25 \mathrm{mV}$, scale bar $5 \mathrm{~nm}$ ). (b) Statistical distribution of the corresponding $d I / d V$ amplitude.

system. For a given eigenstate of a tight-binding-model, it is defined as

$$
\mathrm{NPR}=1 /\left(N \sum_{\vec{r}}|\Psi(\vec{r})|^{4}\right),
$$

where $N$ denotes the number of lattice sites and $\sum_{\vec{r}}|\Psi(\vec{r})|^{2}=$ 1 [33]. The NPR describes the fraction of system sites, which contribute to the spectral weight of an eigenstate. For a completely extended state, which exhibits plane wave characteristics, NPR $\rightarrow 1$. The more localized the state is, the smaller the NPR becomes. For a fully localized state, NPR $\rightarrow 0$. Figure 6 shows the NPR, which was calculated for the Anderson tight-binding model eigenstates of the $s p_{z}$ band as a function of the energy separation from the band onset at $E_{0}=0 \mathrm{meV}$. We also plot the spatially resolved density distribution, $|\Psi(\vec{r})|^{2}$, of selected eigenstates in the insets. $\mathrm{NPR} \approx 0.1$ for the eigenstates near the band onset signals eigenstate localization, which is consistent with the spatial characteristics of the corresponding $|\Psi(\vec{r})|^{2}$. Moving inside the band, the NPR increases, until it plateaus at NPR $\approx 0.9$ deep inside the $s p_{\mathrm{z}}$ band, a trend which is also reflected in the real space characteristics of the $|\Psi(\vec{r})|^{2}$.

\section{APPENDIX E: QUASIPARTICLE LIFETIME EFFECTS}

To illustrate the effect of quasiparticle scattering on the real space characteristics of the LDOS away from Fermi energy, we have recorded a $\mathrm{dI} / \mathrm{dV}$ map near the $m_{\mathrm{j}}=3 / 2$-band edge at $E=1 \mathrm{eV}$ shown in Fig. 7(a). The corresponding LDOS distribution histogram is shown in Fig. 7(b). It is slightly asymmetric, but its characteristics can be well described by a Gaussian distribution, indicating the absence of multifractal scaling of the eigenstate real space distribution.
[1] K. R. Sreenivasan, Fractals and multifractals in fluid turbulence, Annu. Rev. Fluid Mech. 23, 539 (1991).

[2] H. E. Stanley and P. Meakin, Multifractal phenomena in physics and chemistry, Nature (London) 335, 405 (1988).

[3] E. Abrahams, P. W. Anderson, D. C. Licciardello, and T. V. Ramakrishnan, Scaling Theory of Localization: Absence of
Quantum Diffusion in Two Dimensions, Phys. Rev. Lett. 42, 673 (1979).

[4] F. Wegner, The mobility edge problem: continuous symmetry and a conjecture, Z. Phys. B 35, 207 (1979).

[5] P. W. Anderson, Absence of diffusion in certain random lattices, Phys. Rev. 109, 1492 (1958). 
[6] C. Castellani and L. Peliti, Multifractal wavefunction at the localisation threshold, J. Phys. A. 19, L429 (1986).

[7] I. V. Lerner, Distribution functions of current density and local density of states in disordered quantum conductors, Phys. Lett. A 133, 253 (1988).

[8] A. D. Mirlin and Y. V. Fyodorov, Distribution of Local Densities of States, Order Parameter Function, and Critical Behavior Near the Anderson Transition, Phys. Rev. Lett. 72, 526 (1994).

[9] T. C. Halsey, M. H. Jensen, L. P. Kadanoff, I. Procaccia, and B. I. Shraiman, Fractal measures and their singularities: The characterization of strange sets, Phys. Rev. A 33, 1141 (1986).

[10] F. Evers and A. D. Mirlin, Anderson transitions, Rev. Mod. Phys. 80, 1355 (2008).

[11] B. B. Mandelbrot, Intermittent turbulence in self-similar cascades: divergence of high moments and dimension of the carrier, J. Fluid Mech. 62, 331 (1974).

[12] H. G. E. Hentschel and I. Procaccia, The infinite number of generalized dimensions of fractals and strange attractors, Physica D 8, 435 (1983).

[13] M. Morgenstern, J. Klijn, Chr. Meyer, and R. Wiesendanger, Real-Space Observation of Drift States in a Two-Dimensional Electron System at High Magnetic Fields, Phys. Rev. Lett. 90, 056804 (2003).

[14] K. Hashimoto, C. Sohrmann, J. Wiebe, T. Inaoka, F. Meier, Y. Hirayama, R. A. Römer, R. Wiesendanger, and M. Morgenstern, Quantum Hall Transition in Real Space: From Localized to Extended States, Phys. Rev. Lett. 101, 256802 (2008).

[15] A. Richardella, P. Roushan, S. Mack, B. Zhou, D. A. Huse, D. D. Awschalom, and A. Yazdani, Visualizing critical correlations near the metal-insulator transition in $\mathrm{Ga}_{1-x} \mathrm{Mn}_{x} \mathrm{As}$, Science 327, 665 (2010).

[16] K. Zhao, H. Lin, X. Xiao, W. Huang, W. Yao, M. Yan, Y. Xing, Q. Zhang, Z.-X. Li, S. Hoshino et al., Disorder-induced multifractal superconductivity in monolayer niobium dichalcogenides, Nat. Phys. 15, 904 (2019).

[17] M. M. Ugeda, A. J. Bradley, Y. Zhang, S. Onishi, Y. Chen, W. Ruan, C. Ojeda-Aristizabal, H. Ryu, M. T. Edmonds, H.Z. Tsai et al., Characterization of collective ground states in single-layer nbse ${ }_{2}$, Nat. Phys. 12, 92 (2016).

[18] G. Bihlmayer, S. Blügel, and E. V. Chulkov, Enhanced Rashba spin-orbit splitting in $\mathrm{Bi} / \mathrm{Ag}(111)$ and $\mathrm{Pb} / \mathrm{Ag}$ (111) surface alloys from first principles, Phys. Rev. B 75, 195414 (2007).

[19] C. R. Ast, D. Pacilé, L. Moreschini, M. C Falub, M. Papagno, K. Kern, M. Grioni, J. Henk, A. Ernst, S. Ostanin, and P. Bruno, Spin-orbit split two-dimensional electron gas with tunable Rashba and Fermi energy, Phys. Rev. B 77, 081407(R) (2008).

[20] I. Gierz, B. Stadtmüller, J. Vuorinen, M. Lindroos, F. Meier, J. H. Dil, K. Kern, and C. R. Ast, Structural influence on the Rashba-type spin splitting in surface alloys, Phys. Rev. B 81, 245430 (2010).

[21] S. N. P. Wissing, A. B. Schmidt, H. Mirhosseini, J. Henk, C. R. Ast, and M. Donath, Ambiguity of Experimental Spin Information from States with Mixed Orbital Symmetries, Phys. Rev. Lett. 113, 116402 (2014).

[22] C. R. Ast, J. Henk, A. Ernst, L. Moreschini, M. C. Falub, D. Pacilé, P. Bruno, K. Kern, and M. Grioni, Giant Spin Splitting through Surface Alloying, Phys. Rev. Lett. 98, 186807 (2007).
[23] C. R. Ast, G. Wittich, P. Wahl, R. Vogelgesang, D. Pacilé, M. C. Falub, L. Moreschini, M. Papagno, M. Grioni, and K. Kern, Local detection of spin-orbit splitting by scanning tunneling spectroscopy, Phys. Rev. B 75, 201401(R) (2007).

[24] A. L. Efros and B. I. Shklovskii, Coulomb gap and low temperature conductivity of disordered systems, J. Phys. C 8, L49 (1975).

[25] B. L. Altshuler, A. G. Aronov, and P. A. Lee, Interaction Effects in Disordered Fermi Systems in Two Dimensions, Phys. Rev. Lett. 44, 1288 (1980).

[26] Y. Imry and Z. Ovadyahu, Density-of-States Anomalies in a Disordered Conductor: A Tunneling Study, Phys. Rev. Lett. 49, 841 (1982).

[27] E. Bielejec, J. Ruan, and W. Wu, Hard Correlation Gap Observed in Quench-Condensed Ultrathin Beryllium, Phys. Rev. Lett. 87, 036801 (2001).

[28] T. Ando, Numerical study of symmetry effects on localization in two dimensions, Phys. Rev. B 40, 5325 (1989).

[29] A. Mildenberger and F. Evers, Wave function statistics at the symplectic two-dimensional anderson transition: Bulk properties, Phys. Rev. B 75, 041303(R) (2007).

[30] S. Hikami, A. I. Larkin, and Y. Nagaoka, Spin-orbit interaction and magnetoresistance in the two dimensional random system, Prog. Theor. Phys. 63, 707 (1980).

[31] I. M. Lifshitz et al., Energy spectrum structure and quantum states of disordered condensed systems, Sov. Phys. Usp. 7, 549 (1965).

[32] N. F. Mott, Metal-insulator transition, Rev. Mod. Phys. 40, 677 (1968).

[33] $\mathrm{F}$. Wegner, Inverse participation ratio in $2+\varepsilon$ dimensions, $\mathrm{Z}$. Phys. B 36, 209 (1980).

[34] A. Chhabra and R. V. Jensen, Direct Determination of the $\mathrm{f}(\alpha)$ Singularity Spectrum, Phys. Rev. Lett. 62, 1327 (1989).

[35] A. D. Mirlin, Y. V. Fyodorov, A. Mildenberger, and F. Evers, Exact Relations between Multifractal Exponents at the Anderson Transition, Phys. Rev. Lett. 97, 046803 (2006).

[36] C. Rubio-Verdú, A. M. García-García, H. Ryu, D.-J. Choi, J. Zaldívar, S. Tang, B. Fan, Z.-X. Shen, S.-K. Mo, J. I. Pascual, and M. M. Ugeda, Visualization of multifractal superconductivity in a two-dimensional transition metal dichalcogenide in the weak-disorder regime, Nano Lett. 20, 5111 (2020).

[37] M. V. Feigel'man, L. B. Ioffe, V. E. Kravtsov, and E. A Yuzbashyan, Eigenfunction Fractality and Pseudogap State Near the Superconductor-Insulator Transition, Phys. Rev. Lett. 98, 027001 (2007).

[38] M. V. Feigel'man, L. B. Ioffe, V. E. Kravtsov, and E. Cuevas, Fractal superconductivity near localization threshold, Ann. Phys. 325, 1390 (2010).

[39] I. S. Burmistrov, I. V. Gornyi, and A. D. Mirlin, Enhancement of the Critical Temperature of Superconductors by Anderson Localization, Phys. Rev. Lett. 108, 017002 (2012).

[40] L. Dell'Anna, Enhancement of critical temperatures in disordered bipartite lattices, Phys. Rev. B 88, 195139 (2013).

[41] J. Mayoh and A. M. García-García, Global critical temperature in disordered superconductors with weak multifractality, Phys. Rev. B 92, 174526 (2015).

[42] P. Koželj, S. Vrtnik, A. Jelen, S. Jazbec, Z. Jagličić, S. Maiti, M. Feuerbacher, W. Steurer, and J. Dolinšek, Discovery of 
a Superconducting High-Entropy Alloy, Phys. Rev. Lett. 113, 107001 (2014).

[43] L. Sun and R. J. Cava, High-entropy alloy superconductors: Status, opportunities, and challenges, Phys. Rev. Mater. 3, 090301 (2019).

[44] M. Budke, V. Renken, H. Liebl, G. Rangelov, and M. Donath, Inverse photoemission with energy resolution better than 200 mev, Rev. Sci. Instrum. 78, 083903 (2007).

[45] C. Thiede, I. Niehues, A. B. Schmidt, and M. Donath, The acetone bandpass detector for inverse photoemission: operation in proportional and Geiger-Müller modes, Meas. Sci. Technol. 29, 065901 (2018).

[46] A. Zumbülte, A. B. Schmidt, and M. Donath, Momentum resolution in inverse photoemission, Rev. Sci. Instrum. 86, 013908 (2015).

[47] A. Alvermann and H. Fehske, Local distribution approach to disordered binary alloys, Eur. Phys. J. B 48, 295 (2005).

[48] G. Schubert, J. Schleede, K. Byczuk, H. Fehske, and D. Vollhardt, Distribution of the local density of states as a criterion for anderson localization: Numerically exact results for various lattices in two and three dimensions, Phys. Rev. B 81, 155106 (2010).

[49] Y. Asada, K. Slevin, and T. Ohtsuki, Anderson Transition in Two-Dimensional Systems with Spin-Orbit Coupling, Phys. Rev. Lett. 89, 256601 (2002).

[50] W. Ku, T. Berlijn, C.-C. Lee et al., Unfolding First-Principles Band Structures, Phys. Rev. Lett. 104, 216401 (2010).

[51] V. Popescu and A. Zunger, Effective Band Structure of Random Alloys, Phys. Rev. Lett. 104, 236403 (2010).

[52] T. B. Boykin and G. Klimeck, Practical application of zonefolding concepts in tight-binding calculations, Phys. Rev. B 71, 115215 (2005).

[53] T. B. Boykin, N. Kharche, G. Klimeck, and M. Korkusinski, Approximate bandstructures of semiconductor alloys from tight-binding supercell calculations, J. Phys.: Condens. Matter 19, 036203 (2007).

[54] L. Moreschini, A. Bendounan, H. Bentmann, M. Assig, K. Kern, F. Reinert, J. Henk, C. R. Ast, and M. Grioni, Influence of the substrate on the spin-orbit splitting in surface alloys on (111) noble-metal surfaces, Phys. Rev. B 80, 035438 (2009). 